\title{
Effect of soil moisture stress on the emergence, establishment and productivity of Amaranthus (Amaranthus Cruentus L.) Olufolaji, A. $\mathrm{O}^{1 *}$ Odeleye, F. $\mathrm{O}^{2}$. and Ojo, O.D ${ }^{1}$.
}

\author{
${ }^{1}$ National Horticultural Research Institute (NIHORT), \\ PMB 5432, Idi-Ishin, Ibadan - Nigeria \\ ${ }^{2}$ Department of Crop Protection \& Environmental Biology, \\ University of Ibadan, Nigeria. \\ * Correspondence author: olufolajiadenike@yahoo.com
}

\begin{abstract}
Dietary intake of vegetables is low in Sub-Sahara Africa (SSA) compared to the world's average. This situation is worsened by low water availability for vegetable production especially in the dry season. However, amaranth which can be grown in the different agro-ecologies of Nigeria is a dual purpose crop with edible leaves and seeds rich in essential nutrients, minerals and proteins. The crop is also a drought tolerant C4 plant which has the ability to survive under minimum soil moisture condition, hence the reason for its use in the study. This investigation was therefore conducted to discover the optimum water requirements in a screen house, and evaluation of different moisture stress based on the derived optima in two subsequent field trials. In the screen house and field, water stress treatments varied from: $-6.0,-1.2$ to -0.3 bar (the control). Results in the screen house study showed that optimum soil water potential for amaranth was reached at -1.2 bar, and that variety $\mathrm{NH} 84 / 493$ performed better than the others, in all growth and yield parameters measured. In field trials, bringing the moisture content from dry (-6.0 bar) to -1.5 bar optimum soil water potential favored seedling emergence better than all other treatment combinations. However, treatment combination $-0.3,-1.5$ bar when the soil was initially wet (-0.3 bar) then bringing it to the optimum (-1.5 bar) gave the best seedling establishment and productivity. Variety NH84/493 grown at $-0.3,-1.5$ bar performed better than all other interactions. We therefore recommend -1.5 bar and variety $\mathrm{NH} 84 / 493$ amaranth for the SSA vegetable industry, especially for improving dry season productivity.
\end{abstract}

Keywords: Moisture stress, Emergence, Establishment, Production, Amaranth.

\section{INTRODUCTION}

Amaranth (Amaranthus cruentus L.) is important for its prolific vegetative and grain production, which alleviate poverty especially in sub-Sahara Africa (SSA) (Liu and Stutzel 2002; 2004). It is highly regenerative when cut as well as it increases farmers' income when planted in mixture with other crops (Grubben, 1976; David, 2001). Amaranth has become important in urban and peri-urban vegetable industry, which brings more attention to improving its productivity. The vegetable intake of the Sub- Sahara Africa (SSA) populace is far below the world's WHO and FAO recommendations (WHO, 1993; FAO, 1991; Bhargava, Shikia and Ohri 2006).

Amaranthus, a $\mathrm{C}_{4}$ plant when compared with Celosia, a $\mathrm{C}_{3}$ plant requires higher light intensity in order to attain maximum photosynthesis (Grubben, 1976). Ehleringer and Bjokman (1976) also observed that the net photosynthesis of $\mathrm{C}_{4}$ plant is maximum at full sunshine (provided the temperature is at optimum $30^{\circ}-47^{\circ} \mathrm{C}$ ); whereas the $\mathrm{C}_{3}$ plants reach saturation level at $25-50 \%$ of full sunshine. Amaranthus is a short day plant such that during growth Amaranthus caudatus' sensitivity to inflorescence initiation and consequently early maturity in short days was time specific and occurred about 30 days after sowing, which is termed sensitive period; beyond which two days was sufficient to initiate inflorescence primodia. Whereas, in long days of 18 hours, the same species initiated inflorescence primodia, 60 days after germination.

Hauptil (1977), Anon (1984), reported that the $C_{4}$ plant uses about three-fifth the amount of water a $\mathrm{C}_{3}$ plant uses to produce the same amount of plant materials. Cavagrarno and Jain (1985) studied the effect of water stress on four species of Amaranthus and reported that seed yield per plant showed different rates of decreases due to water stress in 
various species. Also irrespective of the species, optimum seed yield was obtained in medium stressed plants while no stress and highly stressed plants gave reduced seed yield.

Good and viable amaranth seeds usually germinate 3-4 days after sowing under field conditions. However, amaranth exhibits slow and irregular seedling emergence, which might be due to the uneven maturity of the racemes which arise in groups. Apart from the environmental factors such as soil moisture, temperature and day length as enumerated above, the maturity pattern of the seeds could also be responsible for the irregular and poor emergence pattern of the seedlings to the extent that emergence could spread over a period of up to 14 days. Once emergence is accomplished a high percentage seedling establishment could be achieved in 14-21 days after sowing.

Root growth ensuring water supply, vigorous seedlings capable of piercing the soil surface and the ability to begin photosynthesis are vital factors of establishment of seedlings, especially from small seeds. Seedling establishment is defined as the formation of the first primary leaves and the root system (Khatamian, Adedipe and Armrod 1973; Olufolaji and Tayo, 1980; 1989; Garcia, Raes and Jacobsen 2003).

The factor limiting vegetable productivity in SSA is availability of agricultural water especially in the dry season. Although production takes place to some extent in urban and peri-urban areas, the majority of vegetables consumed come from the hinterlands where agricultural water is scarce. There is dearth of knowledge about water requirements of amaranth especially as it relates to varietal water use efficiencies (Romo and Haferkamp 1987; Liu and Stutzel 2004; Mwale, Azam-Ali and Massawe 2007). Amaranth varieties differ in morphology, physiological structures, root system and days to maturity. These differences could be responsible for the ability of one variety to use irrigation water more optimally than the other (Olufolaji and Tayo 1980; 1989).

This study therefore explores the effects of variations in moisture stresses among amaranth varieties both in the screen house and field experiments. This was carried out in order to elucidate the optimal water requirements and the variety that optimizes irrigation water usage.

\section{MATERIALS \& METHODS}

A. Screen Trial: The screen house study carried out involved the use of 144 pots each spaced $0.15 \mathrm{~m}$ apart, and each pot is of a 10 liter capacity filled with $8 \mathrm{~kg}$ of $100 \mathrm{~m}$ sand soil. The composition of the soil used is as presented in Table 1.

Seeds were sown, three weeks later seedlings were thinned to 10 per pot. Seedlings were then grown under three conditions of soil moisture potentials: -6.0 bar, - 1.2 bar, and -0.3 bar, equivalent to $7.5 \mathrm{~cm}^{3}$, $10.0 \mathrm{~cm}^{3}$ and $30.0 \mathrm{~cm}^{3}$ volume of irrigation water respectively. The required moisture potential was monitored by gypsum blocks and Tentiometers installed at $0.15 \mathrm{~m}$ (effective root zone of the crops) soil depth. Plants were arranged in a completely randomized block design with four replications. Harvests were carried out at 8 weeks (optimal vegetative stage) and 16 weeks after sowing (optimal seed maturity stage).

B. Field Trial: The soil used was analysed physically and chemically. The physical and chemical characteristics of the soil used are as shown in Table 1. Soil samples taken were air-dried for 5 days under ambient temperature, ground, sieved through a $2 \mathrm{~mm}$ sieve, and analyzed for their various physicochemical properties including total nitrogen, $\mathrm{pH}$, organic carbon, available $\mathrm{P}$, exchangeable $\mathrm{Ca}, \mathrm{K}$, $\mathrm{Mg}, \mathrm{Na}, \mathrm{Fe}, \mathrm{Mn}$ and $\mathrm{Al}$ (Table 1). The procedures used were according to IITA (1976). Total nitrogen and organic carbon were determined by the Kjeldahl (Bremer, 1965) and Walkey and Black procedures (Nelson and Sommers, 1982), procedures respectively. Soil pH was measured using a 1:2 (W/V) soil water suspension ratio. Available phosphorus was analyzed by the Bray P-I method (Bray and Kurtz, 1945). Potassium, Ca and Mg were first extracted using neutral normal NI-Li OAc (IITA, 1976). Thereafter, $K$ was determined by flame emission in the Perkin-Elmer 5000 Atomic absorption spectrophotometer; $\mathrm{Mg}$ and $\mathrm{Ca}$ by atomic absorption; other exchangeable bases according to Jackson, 1958; and CEC was determined as the sum of exchangeable bases and exchangeable acidity (Juo and Fox, 1977). The mechanical analysis was carried out by the hydrometer method (Bouyoucous, 1951). 
Table 1:The physical and chemical characteristics of the soil used for the experiments

\begin{tabular}{|l|l|l|}
\hline \multicolumn{2}{|l|}{ First trial } & Second Trial \\
\hline Textural Classification & 88.6 & 86.6 \\
\hline Particle size distribution (\%) & 5.4 & 6.4 \\
\hline Sand 200cm - 60mm & 6.0 & 1.32 \\
\hline Silt 60mm - 2mm & 1.40 & 1.77 \\
\hline Clay < 2mm & & 0.12 \\
\hline Bulk density (Mg n-T ${ }^{3}$ ) & 2.73 & 115.74 \\
\hline Chemical proportion & 0.14 & 3.36 \\
\hline Organic matter (\%) & 112.53 & 6.50 \\
\hline Total N (\%) & 3.88 & 3.00 \\
\hline Available P (ppm) & 5.25 & 0.65 \\
\hline Exchangeable K me/100g & 3.67 & 13.71 \\
\hline Ca & 1.83 & 98.54 \\
\hline Mg & 15.03 & 6.6 \\
\hline Exchangeable Na me/100g & 97.34 & \\
\hline Cation exchange capacity me/lOOg & 6.1 & \\
\hline Percent base saturation & & \\
\hline pH in H20 & & \\
\hline
\end{tabular}

The soil field capacity was $27.4 \%$ and the permanent wilting point was $3.1 \%$. The treatments imposed were the soil moisture status of the seedbed at sowing, and at subsequent stages plant growth of 8 and 16 WAS. There were four treatments:

(i) An initial dry (-6.0 bar) soil moisture potential when the seeds were sown $(7.5 \mathrm{~cm}$ soil moisture content) and subsequently plots were maintained at $12.0 \mathrm{~cm}^{3}$ moisture content $(-1.5$ bar). Irrigation commenced one week after sowing and was repeated whenever the soil moisture content was less or equal to $12.0 \mathrm{~cm}^{3}$ (-1.5 bar). The plants required about 84 hours to bring soil water potential from $30 \mathrm{~cm}^{3}$ to $100 \mathrm{~cm}^{-}$ ${ }^{3}$ (-1.5 bar).

(ii) An initial wet (-0.3 bar) soil moisture potential in which plots were watered to field capacity before sowing. Subsequently, irrigation was carried out when the soil moisture content was less or equal to $12.0 \mathrm{~cm}^{3}$ (-1.5 bar).

(iii) An initial dry (-6.0 bar) soil moisture potential when the seeds were sown and subsequently plots were maintained at $30.0 \mathrm{~cm}^{3}(-0.3$ bar) soil moisture content. Irrigation commenced one week after sowing and was repeated whenever the soil moisture content was $30.0 \mathrm{~cm}^{3}$ ( -0.3 bar) or less. The plants required about 24 hours to reduce soil water content from $30 \mathrm{~cm}^{3}$ to 27.4 $\mathrm{cm}^{3}$ (-0.3 bar).

(iv) An initial wet (-0.3 bar) soil moisture potential in which plots were watered to field capacity before sowing. Subsequently, irrigation was carried out when the soil moisture content was less than or equal to $30.0 \mathrm{~cm}^{3}$ (-0.3 bar).

This study lasted with harvesting of plants till 16 weeks after sowing, the optimal seed developmental stage after which seed shattering and seed losses set in (Olufolaji and Tayo 1980; 1989). Thereafter the plants were uprooted and analyzed for the number of plants, stem height, number of leaves, leaf area, total plant and leaf fresh and dry mass per plant. Where no significant differences were observed data of the field trial were pooled. The field experiment was a randomized complete block design with four replicates.

The gypsum blocks and the $30 \mathrm{~cm}$ tensiometers (manufactured by Soil Test Incorporation, Santa Barbara, U.S.A.) used were soaked in water for one hour. Thereafter, the gypsum blocks and tensiometers were inserted into $15 \mathrm{~cm}$ deep holes in the wet soil and the soil was placed firmly round them to ensure good contact between the moisture indicators and the soil. The instruments were calibrated by taking soil samples for moisture determination at known soil water potential values. Moisture content was determined gravimetrically by drying soils of known weight at $105^{\circ} \mathrm{C}$ to constant 
weight.

\section{RESULTS}

A. Greenhouse Trial: At 8 weeks, the stem height and leaf area were highest in NH84/493 and lowest in ED82/1040A. At 16 wks NHAc3 had significantly higher number of leaves than NH84/493. Soil moisture maintained at -1.2 bar gave the greatest vegetative plant growth, while the lowest was obtained at -0.3 bar (F.C) (Table 2). The data collected at the peak of vegetative stage (8 weeks) and peak of reproductive stage (16 weeks) after sowing were used to illustrate the effect of interactions. Variety $\mathrm{NH} 84 / 493$ kept at -1.2 bar gave

Table 2: Stein Height (SH cm/plant), Number of leaves (NL) and Leaf Area (LA cur/plant) per plant of three varieties of Amaranthus cruentus sown in the green-house, at different soil moisture potentials (SMP)

\begin{tabular}{|c|c|c|c|c|c|c|}
\hline Treatment Varieties & $\begin{array}{l}\text { SH(cm) } \\
8 \text { weeks }\end{array}$ & $\begin{array}{l}\text { SH (cm) } \\
\text { I } 6 \text { Weeks }\end{array}$ & $\begin{array}{l}\text { NL } 8 \\
\text { Weeks }\end{array}$ & $\begin{array}{l}\text { NL } \\
16 \text { Weeks }\end{array}$ & $\begin{array}{l}\left.\text { LA(cm }{ }^{2}\right) 8 \\
\text { Weeks }\end{array}$ & $\begin{array}{l}\mathrm{LA}\left(\mathrm{cm}^{2}\right) \\
16 \text { Weeks }\end{array}$ \\
\hline N1184/493 & $38.0 a$ & $68.9 a$ & $16.2 \mathrm{a}$ & $24.9 \mathrm{~b}$ & $275 a$ & $472 b$ \\
\hline NHAC3 & $19.3 b$ & $48.3 c$ & $13.38^{\prime}$ & $35.4 a$ & 119.b & $561 a$ \\
\hline HU82/1040 & $16.8 \mathrm{~b}$ & $56.9 b$ & $10.9 \mathrm{c}$ & $32.6 a$ & $100 . c$ & $397 c$ \\
\hline SD & 3.32 & 2.79 & 1.00 & 4.49 & 10.6 & 37.0 \\
\hline $\mathrm{CV}$ & 7,79 & 2.78 & 4.26 & 8.38 & 3.72 & 4.49 \\
\hline SE & 0.96 & 0.81 & 0.29 & 1.30 & 3.07 & 10.7 \\
\hline $\mathrm{I} . \mathrm{SD}(\mathrm{P}=0.05)$ & 3.33 & 2.79 & 0.99 & 4.50 & 10.6 & 37.1 \\
\hline \multicolumn{7}{|l|}{ Soil Moisture Potentials } \\
\hline$-6.0 \mathrm{bar}$ & $21.4 \mathrm{c}$ & $52.6 b$ & $13.1 \mathrm{~b}$ & $32.4 \mathrm{~b}$ & $138.7 \mathrm{~b}$ & $471 a$ \\
\hline$-1.2 \mathrm{bar}$ & $27.6 \mathrm{a}$ & $71.0 a$ & 16.la & $39.5 a$ & $246.8 a$ & $576 a$ \\
\hline$-0.3 \mathrm{bar}$ & 25. Ib & $50.4 b$ & $11.3 c$ & $20.9 c$ & $109.2 \mathrm{c}$ & $383 c$ \\
\hline SD & 2.85 & 7.4 & 1.49 & 4.68 & 28.12 & 49.6 \\
\hline $\mathrm{CV}$ & 11.53 & 12.81 & 11.06 & 15.14 & 17.06 & 10.4 \\
\hline SE & 0.82 & 2.15 & 0.43 & 1.35 & 8.12 & 14.3 \\
\hline LSD (P- 0,05) & 2.45 & 6,38 & 1.28 & 4.02 & 24.16 & 42.7 \\
\hline \multicolumn{7}{|l|}{ Interactions } \\
\hline -6.0bar NH 84/493 & $39.2 \mathrm{~b}$ & $69.1 \mathrm{~b}$ & $15.9 \mathrm{c}$ & $28.4 \mathrm{~cd}$ & $255 b$ & $410 c$ \\
\hline$-1.2 \mathrm{bar} N \mathrm{NH} 84 / 493$ & 46.la & $83.7 \mathrm{a}$ & $20.8 b$ & 33.Ib & $442 a$ & $599 b$ \\
\hline-0.3 bar $\mathrm{NH} 84 / 493$ & $28.8 \mathrm{c}$ & $53.8 d$ & $11.8 \mathrm{~d}$ & 13.le & $126 \mathrm{c}$ & $407 c$ \\
\hline-6.0 har NHAc3 & $12.2 \mathrm{e}$ & $42.2 \mathrm{ef}$ & $\cdot 41.2 \mathrm{a}$ & $39.4 a b$ & 86.d0 & $547 b$ \\
\hline -1.2bar NHAc3 & $17.3 d$ & $63.2 \mathrm{c}$ & $14,9 \mathrm{c}$ & $45.3 a$ & $159 e$ & $694 a$ \\
\hline -0.3bar NHAc3 & $28.4 \mathrm{c}$ & $39.5 f$ & $10.9 d$ & $21.5 d$ & $114 d$ & $438 c$ \\
\hline -6.0bar ED82/1040 & $12.8 \mathrm{e}$ & $46.5 e$ & 9.70 & $29.3 c$ & $75 . \mathrm{Od}$ & $454 c$ \\
\hline -1.2bai- ED82/1040 & $19.4 d$ & $66.2 b$ & $12.4 d$ & $40.3 \mathrm{~cd}$ & $138 \mathrm{c}$ & $434 \mathrm{c}$ \\
\hline-0.3 bar-ED82/1040 & $18.2 \mathrm{~d}$ & $57.9 d$ & $11.3 d$ & 28.1 & $87 . d 5$ & $300 d$ \\
\hline $\mathrm{SD}$ & 2.85 & 7.43 & 1.49 & 4.68 & 28.1 & 49.6 \\
\hline SK & 1.42 & 3.72 & 0.75 & 2.34 & 14.1 & 24.8 \\
\hline LSD $(P=0.05)$ & 4.24 & 111 & ??"' & 6.97 & 41.8 & 73.8 \\
\hline
\end{tabular}

Column means followed by unlike/letters) are significantly different at $(P=-0.05)$ significantly taller plants than all other variety $x$ soil moisture stress treatment combinations. At 8 weeks, the number of leaves was highest in NH84/493 maintained at -1.2 bar soil moisture potential. At 16 weeks, NHAc3 and ED82/1040A had similar but significantly higher number of leaves than all the other variety by soil moisture stress treatment interactions. At 8 weeks, NH84/493 had significantly higher leaf area at -1.2 bars, while at 16 weeks NHAc3 maintained at -1.2 bar gave the highest leaf area (Table 2). These results therefore indicate that moisture stress significantly affect leaf area growth and development in amaranth. 
Agric. Biol. J. N. Am., 2010, 1(6): 1169-1181

Table 3:Total Fresh Weight (TFW g/plant) and Leaf Fresh Weight (LEW g/plant) of three varieties of Amaranthus cruentus sown in the green house, at different soil moisture potentials (SMP)

\begin{tabular}{|c|c|c|c|c|}
\hline & TFW : & TFW & LFW & LFW \\
\hline Treatment Varieties & (g/plant) & (g/plant) & (g/plant) & (g/plant) \\
\hline & 8 Weeks & 16 Weeks & 8 Weeks & 16 Weeks \\
\hline $\mathrm{NH} 84 / 493$ & $19.9 a$ & $63.8 a$ & $2.07 \mathrm{~b}$ & $21.8 \mathrm{a}$ \\
\hline NHAc3 & $5.8 \mathrm{~b}$ & $52.6 \mathrm{~b}$ & $2.37 a$ & $19.7 a b$ \\
\hline ED82/1040A & $5.80 \mathrm{~b}$ & $46.7 \mathrm{c}$ & $2.32 a$ & ; 17.5b \\
\hline SD & 0.95 & 5,54 & 0.45 & 2.54 \\
\hline CV & 5.24 & 5.87 & 11.8 & 7,47 \\
\hline $\mathrm{SH}$ & 0.27 & 1.60 & 0.13 & 0.73 \\
\hline LSD $(P=0.05)$ & 0.95 & 5.54 & 0.45 & 2.54 \\
\hline \multicolumn{5}{|l|}{$\begin{array}{l}\text { Soil Moisture } \\
\text { Potentials }\end{array}$} \\
\hline$-6.0 b a r$ & $8.98 \mathrm{~b}$ & $44.9 \mathrm{~b}$ & $2.49 a$ & $17,9 b$ \\
\hline$-1.2 \mathrm{bar}$ & 15.la & $71.4 \mathrm{a}$ & $2.51 \mathrm{a}$ & $24.8 \mathrm{a}$ \\
\hline$-0.3 \mathrm{bar}$ & $7.35 b$ & $47.2 \mathrm{~b}$ & $1.74 b$ & $16.4 b$ \\
\hline SI) & 1.83 & 6.46 & 0.35 & 2.90 \\
\hline $\mathrm{CV}$ & 17.5 & 119 & 15.9 & 14.7 \\
\hline SE & 0.52 & 1.86 & 0.10 & 0.83 \\
\hline LSD(P- 0=05) & 1.57 & 5.55 & 0.30 &, 2.49 \\
\hline \multicolumn{5}{|l|}{ Interactions } \\
\hline-6.0 barNH84/493 & $19 . \mathrm{Ob}$ & $54,5 b$ & $2.22 \mathrm{bc}$ & $18.8 \mathrm{cde}$ \\
\hline -1.2bar N1184/493 & $30.8 \mathrm{a}$ & $79.6 a$ & $1.99 \mathrm{bcd}$ & $28.3 a$ \\
\hline-0.3 bar NH84/493 & $9.74 \mathrm{c}$ & $57.4 b$ & $1.98 \mathrm{bcd}$ & 18.1cde \\
\hline -6.0barNHAc3 & $4.44 \mathrm{e}$ & $49.8 b$ & 2.21 be & 18.4cde \\
\hline-1.2 barNHAc3 & '6.83cde & $67.9 \mathrm{~b}$ & $3.13 a$ & $24.6 a b$ \\
\hline -0.3bar NHAc3 & 6.11def & $41.3 \mathrm{~b}$ & $1.74 \mathrm{~cd}$ & 16.3de \\
\hline-6.0 barEU82/1040 & $3.51 \mathrm{f}$ & $30.3 c$ & $3.05 a$ & 16.4de \\
\hline -1.2barED82/1040 & $7.69 \mathrm{~cd}$ & $66.7 \mathrm{~b} "$ & $2.40 \mathrm{~b}$ & $21.6 \mathrm{bc}$ \\
\hline$-0.3 \mathrm{barED} 82 / 1040$ & $6.20 \mathrm{~d}$ & $43.0 b$ & $1.51 \mathrm{~d}$ & $14.7 \mathrm{e}$ \\
\hline SD & 1.83 & 6.46 & 0.35 & 2.90 \\
\hline SE & 0.19 & 3,23 & 0.17 & 1.45 \\
\hline $\operatorname{LSD}(P=0.05)$ & 272 & 9.61 & 0.53 & 4.32 \\
\hline
\end{tabular}

Column means followed by unlike/letters) are significantly different at ( $P=-0.05)$ 
Agric. Biol. J. N. Am., 2010, 1(6): 1169-1181

Table 4: :Total Dry Mass (TDM) and Leaf Dry Mass (LDM) per plant of three varieties of Amaranthus cruentus sown in the green house, at different soil moisture

\begin{tabular}{|c|c|c|c|c|}
\hline \multicolumn{4}{|c|}{$\begin{array}{l}\text { Potentials SMP } \\
\text { (SMP). }\end{array}$} & \multirow[b]{2}{*}{$\begin{array}{c}\text { LDM } \\
\text { (g/plant) } \\
16 \text { Weeks }\end{array}$} \\
\hline $\begin{array}{c}\text { Treatment } \\
\text { Varieties }\end{array}$ & $\begin{array}{c}\text { TDM } \\
\text { (g/plant) } \\
8 \text { Weeks }\end{array}$ & $\begin{array}{c}\text { TDM } \\
\text { (g/plant) } \\
16 \text { Weeks }\end{array}$ & $\begin{array}{c}\text { LDM } \\
\text { (g/plant) } \\
8 \text { Weeks }\end{array}$ & \\
\hline N1184/493 & $2.12 \mathrm{a}$ & $5.93 a$ & $0.70 \mathrm{a}$ & $1.93 a$ \\
\hline NHAc3 & $0.70 \mathrm{~b}$ & $4.75 b$ & $0,34 b$ & $2.06 a$ \\
\hline ED82/1040 & $0.60 \mathrm{~b}$ & $4.94 \mathrm{~b}$ & $0.34 \mathrm{~b}$ & $2.11 a$ \\
\hline SD & 0.18 & 0.61 & 0.09 & 0.49 \\
\hline $\mathrm{CV}$ & 8.99 & 6.76 & 9.65 & 14.12 \\
\hline SR & 0.02 & 0.18 & 0.03 & 0.14 \\
\hline $\operatorname{LSD}\left(\mathrm{P}^{=} 0.05\right)$ & 0.18 & 0.61 & 0.09 & 0.49 \\
\hline \multicolumn{5}{|l|}{ Soil Moisture } \\
\hline \multicolumn{5}{|l|}{ Potentials } \\
\hline$-6.0 \mathrm{bar}$ & $1.07 \mathrm{~b}$ & $5.05 b$ & $0.45 b$ & $2.02 a$ \\
\hline$-1.2 \mathrm{bar}$ & $1.47 a$ & $6.42 a$ & $0.82 a$ & $2.01 \mathrm{a}$ \\
\hline$-0.3 \mathrm{bar}$ & $0.88 \mathrm{c}$ & $4.15 \mathrm{c}$ & $0.39 a$ & $1,72 b$ \\
\hline SD & 0.10 & 0.47 & 0.11 & 0,37 \\
\hline $\mathrm{CV}$ & 8,95 & 9.03 & 20.2 & 18.4 \\
\hline $\mathrm{SE}$ & 0.03 & 0,14 & 0.03 & 0.11 \\
\hline LSD $(P=0.05)$ & 0.09 & 0.40 & 0.09 & 0.32 \\
\hline \multicolumn{5}{|l|}{ Interactions } \\
\hline-6.0 barNH83/493 & $2.32 b$ & $6.08 \mathrm{~b}$ & $0.82 \mathrm{~b}$ & $1.83 b$ \\
\hline -1.2barNH84/493 & $2.92 a-$ & $7.48 a$ & $1.56 \mathrm{a}$ & $1.70 \mathrm{~b}$ \\
\hline-0.3 bar NH84/493 & $1.10 \mathrm{c}$ & $4,21 \mathrm{e}$ & $0.51 \mathrm{c}$ & $2.24 b$ \\
\hline -6.0bar NHAc3 & $0.49 f$ & $5.21 \mathrm{c}$ & $0.21 d$ & $2.4 a b$ \\
\hline$-1.2 \mathrm{barNHAc3}$ & $0.75 \mathrm{de}$ & $5.03 c^{\prime \prime}$ & $0.44 \mathrm{~cd}$ & $1.96 \mathrm{~b}$ \\
\hline$-0.3 \mathrm{bar} \mathrm{NHAc3}$ & $0.87 d$ & $4,02 d$ & $0.36 \mathrm{~cd}$ & $1.80 \mathrm{~b}$ \\
\hline-6.0 barED82/1040 & $0.37 f$ & $3.85 d$ & $0.30 \mathrm{~d}$ & $2.34 a b$ \\
\hline -1.2bai-ED82/1040 & $0.74 \mathrm{de}$ & $6.75 b$ & $0.44 \mathrm{c}$ & $2.85 a$ \\
\hline-0.3 barED82/1040 & $0.68 \mathrm{e}$ & $4.21 d$ & $0.27 d$ & $1.11 \mathrm{c}$ \\
\hline SD & 0.10 & 0.46 & 0.11 & 0.37 \\
\hline $\mathrm{SL}$ & 0,05 & 0.20 & 0.05 & 0.18 \\
\hline $\operatorname{LSD}(P=0.05)$ & 0.15 & 0.69 & 0.16 & 0.55 \\
\hline
\end{tabular}

Column means followed by unlike/letters) are significantly different at $P \wedge 0.05$ 
Agric. Biol. J. N. Am., 2010, 1(6): 1169-1181

Table 5: Seedling Emergence (per $\mathrm{m}^{2}$ ) and Number of Plants (per $\mathrm{m}^{2}$ ) of Amanratus cruentus L. sown in the Field, at different soil moisture potentials (SMP).

\begin{tabular}{|c|c|c|}
\hline $\begin{array}{l}\text { Treatment } \\
\text { Varieties }\end{array}$ & $\begin{array}{c}\text { Emergence } \\
\text { No. of seedlings }\left(\text { per } \mathrm{m}^{2}\right)\end{array}$ & No. of Plants (Plants $/ \mathrm{m}^{2}$ ) \\
\hline $\begin{array}{l}\mathrm{NH84/493} \\
\mathrm{NHAc3} .\end{array}$ & $\begin{array}{l}1386 a \\
1469 a\end{array}$ & $\begin{array}{l}35 \mathrm{sb} \\
439 \mathrm{a}\end{array}$ \\
\hline ED82/1040 & $1538 a$ & $432 a$ \\
\hline SD & 2364 & 39.9 \\
\hline $\mathrm{CV}$ & 8.07 & 4.87 \\
\hline $\mathrm{SH}$ & 59.1 & 9.97 \\
\hline $\operatorname{LSD}(\mathrm{P}=0,05)$ & 205 & 34.6 \\
\hline \multicolumn{3}{|l|}{ Soil Moisture Potentials } \\
\hline$-6.0 .-1.5 \mathrm{bar}$ & $1963 a$ & $673 a$ \\
\hline$-0.3,-1.5 \mathrm{bar}$ & $1402 b$ & $500 \mathrm{~b}$ \\
\hline$-6.0,-0.3 \mathrm{bar}$ & 1042c & $238 \mathrm{c}$ \\
\hline$-0.3,-0.3 \mathrm{bar}$ & $145 b$ & $224 c$ \\
\hline SD & 275 & 59.3 \\
\hline $\mathrm{CV}$ & 18.73 & 14.5 \\
\hline SE & 79.23 & 17.1 \\
\hline $\operatorname{LSD}(\mathrm{P}=0.05)$ & 230.30 & 49.8 \\
\hline \multicolumn{3}{|l|}{ Interactions } \\
\hline $\begin{array}{l}\text {-6.0. -1.5barNH/493 } \\
\text {-0.3.-1.5barNH/493 }\end{array}$ & $\begin{array}{l}1567 \mathrm{bc} \\
1170 \mathrm{~cd}\end{array}$ & $\begin{array}{l}398 c d \\
436 c\end{array}$ \\
\hline$-6,0 .-0.3$ barNH/493 & $1289 c$ & 318de \\
\hline -0.3.-0.3barNH/493 & $1518 \mathrm{bc}$ & $270 \mathrm{e}$ \\
\hline -6.0,-1.5barNHAc3 & $1875 \mathrm{~b}$ & $816 a$ \\
\hline -0.3.-1.5barNHAc3 & $1405 c$ & $482 \mathrm{c}$ \\
\hline -6.0.-0.3barNHAc3 & $1048 \mathrm{~cd}$ & 240ed \\
\hline$-0.3,-0,3$ barNHAc3 & $1551 \mathrm{bc}$ & $2201^{\prime}$ \\
\hline -6.0.- .5barED1040 & $2447 a$ & $806 a$ \\
\hline -0.3.- ,5barHD1040 & $1633 b c$ & $584 b$ \\
\hline -6.0.-0.3barED1040 & $789 d$ & $158 f$ \\
\hline -0.3.-0.3barED1040 & 1284 & $183 f$ \\
\hline SD & 274 & 59.3 \\
\hline SE LSD $(P=0.05)$ & $\begin{array}{l}137 \\
398\end{array}$ & $\begin{array}{l}29.6 \\
86.2 \\
\end{array}$ \\
\hline
\end{tabular}

Column means followed by unlike/letter(s) are significantly different at $\mathrm{P}=0.05$ 
Table 6: Stem Height (SH cm), Number of leaves (NL) and Leaf Area $\left(\mathrm{LA} \mathrm{cm}^{2}\right)$ per plant of three Varieties of Amarantus cruentus Sown in the Field, at Different Soil Moisture Potentials (SMP).

\begin{tabular}{|c|c|c|c|c|c|c|}
\hline $\begin{array}{l}\text { Treatment } \\
\text { Varieties }\end{array}$ & $\begin{array}{l}\text { SH(cm) } \\
8 \text { Weeks }\end{array}$ & $\begin{array}{c}\mathrm{SH}(\mathrm{cm}) \\
16 \text { Weeks }\end{array}$ & $\begin{array}{c}\text { NL } \\
8 \text { Weeks }\end{array}$ & $\begin{array}{c}\text { NL } \\
16 \text { Weeks }\end{array}$ & $\begin{array}{l}\text { LA }\left(\mathrm{cm}^{2}\right) \\
8 \text { Weeks }\end{array}$ & $\begin{array}{l}\mathrm{LA}\left(\mathrm{cm}^{2}\right) 16 \\
\text { Weeks }\end{array}$ \\
\hline $\mathrm{NH} 84 / 493$ & $38.9 a$ & $54.7 a$ & $13.3 \mathrm{c}$ & 21.0 & $631 a$ & $14 a$ \\
\hline NHAc3 & $28.4 b$. & $36.7 \mathrm{~b}$ & $20 . b$ & $37.8 \mathrm{~b}$ & $452 b$ & $533 \mathrm{~b}$ \\
\hline ED82/1040A & $27.8 \mathrm{~b}$ & $39 . b$ & $33.6 \mathrm{a}$ & $76.2 \mathrm{a}$ & $268 \mathrm{c}$ & $347 c$ \\
\hline SD & 3.90 & 3.90 & 6.13 & 12.2 & 34.2 & 156 \\
\hline $\mathrm{CV}$ & 6.15 & 4.49 & 13.7 & 1.37 & 3.80 & 11.6 \\
\hline SE & 9.97 & 0.97 & 1.53 & 3.08 & 8.56 & 38.9 \\
\hline LSD $(P=0.05)$ & 3.38 & 3.38 & 5.31 & 107 & 29.7 & 135 \\
\hline \multicolumn{7}{|l|}{ Soil/Moisture } \\
\hline \multicolumn{7}{|l|}{ Potentials } \\
\hline$-6.0,-] .5 \mathrm{bar}$ & $22.7 \mathrm{c}$ & 21.Ic & $17.4 \mathrm{c}$ & $32.8 \mathrm{c}$ & $250 c$ & $178 \mathrm{c}$ \\
\hline$-0.3,-1.5 \mathrm{bar}$ & $28.6 b$ & $45.6 b$ & $14.5 \mathrm{c}$ & $51.2 b$ & 192d & $934 b$ \\
\hline$-6.0,-0.3 \mathrm{bar}$ & $47.8 \mathrm{a}$ & $70.3 a$ & $34.6 \mathrm{a}$ & $67.9 a$ & $107 a$ & $172 a$ \\
\hline$-0.3,-0.3 \mathrm{bar}$ & $27.9 b$ & $28.9 \mathrm{c}$ & $23.3 b$ & 28.Ic & $343 b$ & $385 b$ \\
\hline SD & 3.16 & 8.96 & 5.09 & 12.1 & 66.1 & 183 \\
\hline $\mathrm{CV}$ & 9.96 & 20.6 & 22.69 & 26.9 & 14.7 & 777 \\
\hline SE & 0.91 & 2.58 & 1.46 & 3.5 .0 & 19.1 & 52.7 \\
\hline $\operatorname{LSD}(P=0.05)$ & 2.65 & 7.52 & 4.27 & 10.2 & 55.4 & 153 \\
\hline \multicolumn{7}{|l|}{ Interactions } \\
\hline$-6.0,-1.5$ bar 493 & $37.3 \mathrm{c}$ & $27.5 \mathrm{c}$ & $15.8 \mathrm{~d}$ & $14,6 \mathrm{c}$ & $477 \mathrm{c}$ & $158 \mathrm{c}$ \\
\hline$-0.3,-1.5$ bar 493 & $38.9 \mathrm{c}$ & $61.3 \mathrm{~b}$ & $9.5 d$ & $30.5 c$ & $176 d$ & $723 a$ \\
\hline$-6.0,-0.3$ bar493 & $60.3 a$ & $99.5 a$ & $16.4 \mathrm{~d}$ & $28.8 \mathrm{c}$ & $1578 a$ & $322 a$ \\
\hline$-0.3,-0.3$ bar493 & $9.5 d$ & $30.5 c$ & $11.5 d$ & $10.3 c$ & $292.0 d$ & $439 a$ \\
\hline $\begin{array}{l}-6.0,-1.5 b a r-A c 3 \\
-0.3,-1.5 \text { bar-Ac3 }\end{array}$ & $\begin{array}{l}9.8 d \\
24.6 d\end{array}$ & $30.3 c$ 31.0c & 13.9d 11.Id & $38.3 \mathrm{c} 38.1 \mathrm{c}$ & $\begin{array}{l}189.2 d \\
141.5 d\end{array}$ & $\begin{array}{l}303 b \\
304 b\end{array}$ \\
\hline$-6.0,-0.3 \mathrm{barAc3}$ & $37.0 \mathrm{c}$ & $56.8 \mathrm{~b}$ & $34.0 b$ & $45.7 \mathrm{c}$ & $1030.1 b$ & $125 c$ \\
\hline$-0.3,-0.3$ bar Ac3 & 32. Ic & $28.5 \mathrm{c}$ & $22.8 \mathrm{c}$ & 29.Oc & $447.3 \mathrm{c}$ & $274 b$ \\
\hline$-6.0,-1.5$ bar $1040 \mathrm{~A}$ & $11.0 \mathrm{e}$ & $29.5 \mathrm{c}$ & $22.5 \mathrm{c}$ & $45.5 c$ & $84.7 d$ & $73.8 \mathrm{~d}$ \\
\hline$-0.3,-1.5$ bar 1040A & $22.3 d$ & $44.5 b$ & $23.0 \mathrm{c}$ & $85 . \mathrm{Ob}$ & $259.9 d$ & $173 c$ \\
\hline$-6.0,-0.3$ bar I040A & $46.0 \mathrm{~b}$ & $54.5 b$ & $53.5 \mathrm{a}$ & $129.4 a$ & $439.3 c$ & $698 a$ \\
\hline$-0.3,-0.3$ bar 1040A & $32.0 \mathrm{c}$ & $27.8 \mathrm{c}$ & $35.5 b$ & $45.0 c$ & $288.8 d$ & $441 a$ \\
\hline SD & 3.16 & 8.96 & 5.09 & 12.14 & 66.08 & 183 \\
\hline SE & 1.58 & 4.84 & 2.54 & 6.07 & 33.04 & 91.3 \\
\hline $\operatorname{LSD}(P=0.05)$ & 4.59 & 13.03 & 2.40 & 17.64 & 96.03 & 265 \\
\hline
\end{tabular}

Column means followed by unlike/letter(s) are significantly different at $\mathrm{P}=0.05$

At 8 weeks, the grain amaranth variety NH84/493 gave a significantly higher total plant fresh mass than NHAc3 while the lowest plant fresh mass was recorded in ED82/1040A at 16 weeks. At 8 weeks, the leaf fresh weight was similar in all the varieties studied (Table 3). Moderate moisture stress of -1.2 bar gave significantly highest total fresh and leaf fresh weights compared to all other moisture treatments. This moisture stress ( -1.2 bar) combined with variety $\mathrm{NH84/493}$ performed best compared to all other variety $x$ moisture stress interactions (Table 3).

At 8 weeks, the total plant dry mass was significantly higher in NH84/493 than the varieties NHAC3 and ED82/1040A. At 8 and 16 weeks, the leaf dry mass was similar in all the three varieties. The highest total 
plant dry mass and leaf dry weights occurred in the soil moisture maintained at -1.2 bar (Table 4). At 8 weeks, the leaf fresh mass were similar but significantly better at -1.2 and -0.3 bar than at -6.0 bar soil moisture potential. At 16 weeks, plants maintained at -6.0 bar recorded the least leaf fresh mass, while at 16 weeks plants maintained at -0.3 bar soil moisture potential recorded the least total plant and leaf dry mass. Interactions showed that at 8 weeks the total plant fresh mass, leaf fresh mass and leaf dry mass of NH84/493 were highest at -1.2 bar soil moisture potential. At 16 weeks, the three varieties gave similar but significantly higher, total 4 plant fresh mass, leaf fresh mass and leaf dry mass at -1.2 bars than at -0.3 bar and -6.0 bar soil moisture potential (Tables $3 \& 4$ ).

B. Field Trial: The number of emerged seedlings did not differ significantly among the three varieties tested. Sowing seeds in initially dry soil (ID - 6.0 bar), and subsequently maintaining the soil moisture potential at -1.5 bar (i) gave the highest percent seedling emergence, while sowing seeds in initially dry soil and subsequently maintaining the soil at -0.3 bar (iii) gave the lowest percent emergence (Table 5). Variety ED82/1040A at (i) and (iii) gave the highest and the lowest percent seedling emergence, respectively (Table 5). Varieties NHAc3 and ED82/1040A established similar but significantly higher number of plants than NH84/493. The number of plants established was significantly different among the soil moisture potentials in the order (i) $>$ (ii) $>$ (iii) $>$ (iv). Interactions showed that the number of plants established was highest in NHAc3 exposed to (i) and least with ED82/1040A at (iii) (Table 5).

The number of leaves per plant was similar but significantly higher in the vegetable types $\mathrm{NHAc3}$ and ED82/1040A than the grain type NH84/493 (Table 6). At 8 weeks, the leaf area was significantly higher in the order: NH84/493 > NHAc3 > ED82/1040A (Table 6 ). The stem height, number of leaf and leaf area were greater when the soil moisture was kept at $6.0,-0.3$ bars (iii) than those of other soil moisture potential (Table 6), Variety NH84/493 grew tallest while NHAc3 produced the highest number of leaf and leaf area at (iii) (Table 6).

At 8 weeks after sowing, variety NH84/493 had significantly higher total plant fresh weight than the two vegetable types, while at 16 weeks there were no significant differences in the leaf fresh weight of varieties NHAc3 and ED82/1040A (Table 7). Variety $\mathrm{NH84/493}$ had significantly higher total plant fresh and dry mass than the two vegetable types. $\mathrm{NH} 84 / 493$ and NHAc3 had similar but significantly higher leaf fresh weight than ED82/1040A at 16 weeks. The two vegetable amaranths NHAc3 and ED82/1040A had similar but significantly lower leaf fresh mass than the grain amaranth NH84/493 at 8 weeks after sowing. However, at 16 weeks, the early maturing vegetable amaranth NHAc3 produced the greatest leaf fresh mass than the late maturing variety ED82/1040A and the grain type amaranth

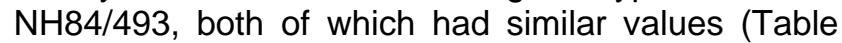
7). At 8 and 16 weeks, the early flowering variety $\mathrm{NH84/493}$ was superior in total fresh mass and total dry matter production than the other varieties (Table 7).

The seed dry weight was understandably highest in the pale-seeded grain type (NH84/493) and least in the black seeded late flowering vegetable type ED82/1040A (Table 8). The seed dry weight was significantly different among the various soil moisture potentials in the order: $-6.0,-0.3$ bars (iii) $>-0.3,-$ 1.5bars (ii) > -0.3. -0.3 bars (iv) $>-6.0 .-1.5$ bars (i) soil moisture potential (Table 8). The interactive effects showed that variety $\mathrm{NH84/493}$ exposed to $6.0,-0.3$ bars (iii) gave the highest seed dry weight (Table 8).

The total fresh weight, leaf fresh weight, leaf dry and seed dry weight were highest at $-6.0,-0.3$ bars (iii) and lowest at 8 weeks $-0.3,-0.3$ bars (iv). Variety $\mathrm{NH84/493}$ at (iii) gave the highest total dry mass and leaf dry mass, while all the varieties kept at (i) at final harvest of 16 weeks gave the least dry matter production (Tables $7 \& 8$ ). Interaction showed that NH84/493 kept at-6.0,-0.3 bars (iii) gave significantly higher plant fresh mass and dry matter production than the other varieties $x$ soil moisture potential combinations (Tables $7 \& 8$ ), 
Agric. Biol. J. N. Am., 2010, 1(6): 1169-1181

Table 7: Total Fresh Mass (TFM) and Leaf Fresh Mass (LFM) grams per plant of three varieties of Amaranhus cruentus sown in the Field, at different soil moisture potentials (SMP).

\begin{tabular}{|c|c|c|c|c|}
\hline $\begin{array}{c}\text { Treatment } \\
\text { Varieties }\end{array}$ & $\begin{array}{c}\text { TFM } \\
\text { (g/plant) } \\
8 \text { Weeks }\end{array}$ & $\begin{array}{c}\text { TFM } \\
\text { (g/plant) } \\
16 \text { Weeks }\end{array}$ & $\begin{array}{c}\text { LFM (g/plant) } 8 \\
\text { Weeks }\end{array}$ & $\begin{array}{c}\text { LFM } \\
\text { (g/plant) } \\
16 \text { Weeks }\end{array}$ \\
\hline $\mathrm{NH84/493}$ & $75.4 a$ & $100 a$ & $21.2 \mathrm{a}$ & $26.6 a$ \\
\hline NHAc3 & $21.5 \mathrm{c}$ & $52.3 \mathrm{c}$ & $6.4 \mathrm{~b}$ & $25.3 a$ \\
\hline ED82/1040 & $26,5 b$ & $69.2 \mathrm{~b}$ & $9.4 \mathrm{~b}$ & $21.4 \mathrm{~b}$ \\
\hline SD & 4.58 & 4.92 & 1.78 & 3.02 \\
\hline $\mathrm{CV}$ & 5.57 & 3.33 & 7.24 & 6.18 \\
\hline SE & 1.14 & 1.23 & 0.44 & 0.75 \\
\hline $\operatorname{LSD}(P=0.05)$ & 3.97 & 4.27 & 1.54 & 2.62 \\
\hline \multicolumn{5}{|l|}{ Soil Moisture } \\
\hline \multicolumn{5}{|l|}{ Potentials } \\
\hline -6.0.-1.5bar & $16.9 \mathrm{c}$ & $39.9 c$ & $5.2 \mathrm{c}$ & $8.4 \mathrm{~d}$ \\
\hline$-0.3 .-1.5 \mathrm{bar}$ & 33.lb & $82.4 \mathrm{~b}$ & $9.6 \mathrm{~b}$ & $23.6 \mathrm{~b}$ \\
\hline -6.0. -0.3bar & $99.9 a$ & $135 a$ & $30.5 a$ & $51.2 a$ \\
\hline$-0.3 .-0.3 \mathrm{bar}$ & $14.5 \mathrm{c}$ & $37.7 \mathrm{c}$ & $3.9 \mathrm{~d}$ & $14.5 \mathrm{c}$ \\
\hline SD & 4.80 & 7.30 & 2.34 & 3.71 \\
\hline $\mathrm{CV}$ & 11.68 & 10.2 & 19.0 & 15.2 \\
\hline SE & 1.38 & 2.16 & 0.67 & 1.07 \\
\hline $\mathrm{LSD}(\mathrm{P}=0.05)$ & 4.03 & 6.29 & 1.96 & 3.11 \\
\hline \multicolumn{5}{|l|}{ Interactions } \\
\hline$-6.0,-1.5$ bar493 & $32.3 d$ & $69.6 e$ & H).3d & $14.3 \mathrm{~cd}$ \\
\hline$-0.3,-1.5 b a r 493$ & $64.9 \mathrm{~h}$ & $112 b$ & $16.3 \mathrm{c}$ & $24.2 \mathrm{~b}$ \\
\hline$-6.0,-0.3$ bar 493 & $188.7 a$ & $191 a$ & $54.2 \mathrm{a}$ & $52.4 a$ \\
\hline$-0.3,-0.3$ bar $4 y 3$ & 15.6ef & $27.5 \mathrm{gh}$ & $4.1 e$ & $15.4 \mathrm{~cd}$ \\
\hline $\begin{array}{l}-6.0,-1.5 \text { barNHAc3 }-0.3,- \\
1.5 \text { barAc3 }\end{array}$ & $\begin{array}{l}8.9 f \\
14.9 \mathrm{ef}\end{array}$ & $\begin{array}{l}26.9 \mathrm{gh} \\
48.4 \mathrm{f}\end{array}$ & $\begin{array}{l}2.6 \mathrm{c} \\
3.9 \mathrm{e}\end{array}$ & $\begin{array}{l}5.6 e \\
28.2 b\end{array}$ \\
\hline -6.0. -0.3barAc3 & $51.6 \mathrm{c}$ & $98.0 \mathrm{c}$ & $16.3 \mathrm{c}$ & $51.9 a$ \\
\hline$-0.3,-0.3$ barAc3 & $10.4 f$ & $35.7 \mathrm{~g}$ & $2.9 \mathrm{e}$ & $15.7 \mathrm{~cd}$ \\
\hline$-6.0 .-1.5$ barl040A & $9.4 f$ & $23.2 \mathrm{~h}$ & $2.9 \mathrm{e}$ & $5.3 e$ \\
\hline$-0.3,-1.5$ bar 1040A & $19.5 \mathrm{e}$ & $86.6 d$ & $8,7 d$ & $18.4 \mathrm{c}$ \\
\hline$-6.0,-0.3$ bar1040A & $59.6 b$ & $117.1 b$ & $21.1 \mathrm{~b}$ & $49.3 a$ \\
\hline$-0.3,-0.3 \mathrm{bar}$ ।040A & $17.5 \mathrm{e}$ & $50.0 f$ & $4.7 e$ & $12.6 \mathrm{~d}$ \\
\hline SD & 4,80 & 7.50 & 2.34 & 3.71 \\
\hline SE & 2.40 & 3.75 & 1.17 & 1.85 \\
\hline $\operatorname{LSD}(P=0.05)$ & 6.98 & 10.90 & 3.40 & 5.39 \\
\hline
\end{tabular}

Column means followed by unlike/letter(s) are significantly different at $\mathrm{P}=0.05$ 
Agric. Biol. J. N. Am., 2010, 1(6): 1169-1181

Table 8.Total Dry Mass (TDM) and Leaf Dry Mass (LDM) grams per Plant of Three Varieties of Amaranthus cruentus Sown in the Field, at different soil moisture potentials (SMP).

\begin{tabular}{|c|c|c|c|c|c|}
\hline $\begin{array}{c}\text { Treatment } \\
\text { Varieties }\end{array}$ & $\begin{array}{c}\text { TDM } \\
\text { (g/plant) } \\
8 \text { Weeks }\end{array}$ & $\begin{array}{c}\text { TDM } \\
\text { (g/plant } \\
16 \text { Weeks }\end{array}$ & $\begin{array}{c}\text { LDM } \\
\text { (g/plant) } \\
8 \text { Weeks }\end{array}$ & $\begin{array}{c}\text { LDM } \\
\text { (g/plant) } 16 \\
\text { Weeks }\end{array}$ & $\begin{array}{c}\text { Seed DM } \\
\text { (g/plant) } \\
16 \text { Weeks }\end{array}$ \\
\hline $\mathrm{NH} 84 / 493$ & $9.19 a$ & $17.13 a$ & $2.12 a$ & $4.58 \mathrm{~b}$ & $3.75 \mathrm{a}$ \\
\hline NHAc3 & $2.27 \mathrm{~b}$ & $11.39 \mathrm{~b}$ & $0.78 \mathrm{c}$ & 5.0la & $2.63 b$ \\
\hline ED 8 2/1040 & $3.18 b$ & $12.44 \mathrm{c}$ & $1.20 \mathrm{~b}$ & $3.89 c$ & $2.02 \mathrm{c}$ \\
\hline SD & 1.11 & 0.44 & 0.08 & 0,26 & 0.36 \\
\hline $\mathrm{CV}$ & 11.37 & 1.62 & 3.27 & 2.96 & 6.42 \\
\hline SE & 0.27 & 0.11 & 0.02 & 0.06 & 0.09 \\
\hline $\operatorname{LSD}(P=0.05)$ & 0.96 & 0.38 & 0.07 & 0.23 & 0.31 \\
\hline \multicolumn{6}{|l|}{ Soil Moisture } \\
\hline \multicolumn{6}{|l|}{ Potentials } \\
\hline$-6.0,-1.5 \mathrm{bar}$ & $2.49 c$ & $6.19 d$ & $0.8 \mathrm{Gc}$ & $1.38 \mathrm{c}$ & $1.04 d$ \\
\hline$-0.3,-1.5 \mathrm{bar}$ & $3.86 \mathrm{~b}$ & $10.39 \mathrm{~b}$ & $1.10 \mathrm{~b}$ & $2.61 b$ & $3.11 \mathrm{~b}$ \\
\hline$-6.0,-0.3 \mathrm{bar}$ & $11.38 a$ & $28.07 a$ & $2.98 a$ & $11.30 \mathrm{a}$ & $4.90 a$ \\
\hline$-0.3,-0.3 \mathrm{bar}$ & $1.79 d$ & $9.95 c$ & $0.60 d$ & $2.70 \mathrm{~b}$ & $2.15 \mathrm{c}$ \\
\hline SD & 1.06 & 0.73 & 0.19 & $0.41 \mathrm{c}$ & 0.53 \\
\hline $\mathrm{CV}$ & 21.89 & 5.41 & 14.20 & 9.27 & 18.95 \\
\hline SE & 0.30 & $0: 21$ & 0.05 & 0.12 & 0.15 \\
\hline $\mathrm{ESD}(\mathrm{P}=0.05)$ & 0.89 & 0.62 & 0.16 & 0.35 & 0.44 \\
\hline $\begin{array}{l}\text { Interactions } \\
-6.0,-1.5 \text { bar493 }\end{array}$ & $4.85 \mathrm{c}$ & $10.23 \mathrm{e}$ & 0.54 & $2.14 \mathrm{e}$ & $2.27 \mathrm{cf}$ \\
\hline$-0.3,-1.5$ bar493 & $6.75 b$ & $-15,8 d$ & $1.79 \mathrm{c}$ & $3.79 d$ & $3.95 b c$ \\
\hline$-6.0 .-0.3$ bar 493 & $22.91 a$ & $35.6 a$ & $4.42 \mathrm{a}$ & $10.2 \mathrm{c}$ & $6.82 \mathrm{a}$ \\
\hline $\begin{array}{l}-0.3,-0.3 \text { bar493 } \\
-6.0,-1.5 \text { barNHAc3 }\end{array}$ & $2.27 \mathrm{~d} 1.36 \mathrm{~d}$ & $6.85 \mathrm{~g} \mathrm{4.68h}$ & $0.74 \mathrm{e} 0.36 \mathrm{f}$ & $\begin{array}{l}2.22 \mathrm{e} \\
0.96 f\end{array}$ & $\begin{array}{l}1.971 \\
0.38 \mathrm{~g}\end{array}$ \\
\hline$-0.3,-1.5$ barNHAc3 & $2.32 \mathrm{~d}$ & $9.23 f$ & $0.42 f$ & $2.50 \mathrm{e}$ & 3.05de \\
\hline$-6.0,-0.3$ barNHAc3 & $4.23 \mathrm{c}$ & $20.1 \mathrm{c}$ & $2.02 \mathrm{c}$ & $12.4 a$ & $4.50 \mathrm{~b}$ \\
\hline -0.3,-0.3barNHAc3 & $1.17 \mathrm{~d}$ & $11,6 \mathrm{e}$ & $0.33 f$ & $4.16 \mathrm{~d}$ & 2.60def \\
\hline$-6.0,-1.5$ bar $1040 \mathrm{~A}$ & $1.27 \mathrm{~d}$ & $3.66 \mathrm{~h}$ & $0.51 \mathrm{ef}$ & $1.05 f$ & $0.47 \mathrm{~g}$ \\
\hline$-0.3,-1.5$ bar $1040 \mathrm{~A}$ & $2.50 \mathrm{~d}$ & $6.19 \mathrm{~g}$ & $1.08 \mathrm{~d}$ & $1.53 f$ & $2.35 \mathrm{ef}$ \\
\hline$-6.0,-0.3$ bar $1040 \mathrm{~A}$ & $7 . \mathrm{Ob}$ & $28.5 b$ & $2.49 b$ & $11.3 b$ & $3.37 \mathrm{~cd}$ \\
\hline$-0.3,-0.3$ bar I040A & $1.9 \mathrm{~d}$ & $11.4 \mathrm{e}$ & $0.74 \mathrm{e}$ & $1.72 \mathrm{f}$ & 1.90 \\
\hline SD & 1.06 & 0.73 & 0.19 & 0.41 & 0.53 \\
\hline SE & 0.53 & 0.36 & 0.09 & 0.20 & 0.26 \\
\hline $\operatorname{LSD}\left(P^{=} 0.05\right)$ & 1.55 & 1.07 & 0.28 & 0.60 & 0.77 \\
\hline
\end{tabular}

Column means followed by unlike/letter(s) are significantly different at $\mathrm{P}=0.05$

\section{DISCUSSION}

The results obtained in this study indicate that in all the trials, though the imposed treatments had significant effects on the plant characteristics of amaranth, soil moisture potential was more significant than varietal effect. This conclusion was clarified by the fact that growth and development of the three varieties of amaranth were similar, thus the soil moisture effect was more important than the varietal effect.

Seedling emergence (in the greenhouse) was adversely affected by the soil moisture potential of 0.3 bar. Although equal numbers of seeds were sown in each treatment, the low moisture potential 
mitigated against effective emergence and establishment of the seedlings, so that fewer than the expected number of seedlings were established. Grubben (1976) similarly observed less emergence and establishment during the rainy season under field conditions in Dahomey. He attributed this in part to excessive moisture with possible interference with soil aeration, reduction in mineral salts due to leaching and possible effect of reduced sunshine due to prolonged overcast.

In amaranthus, initial maintenance of soil moisture of -6.0 bar must have been adequate for seed germination and seedling emergence. There was significant increase in emergence after two weeks in seedlings that were not watered for one week compared with those initially wet followed by moderate stress or no stress conditions. The higher emergence value at the initially high stress followed by moderate stress condition compared with the other treatments, might be due to series of wetting and drying pulses attendant upon initial period (one week) of stress imposed at the moderate stress of 1.5 bar.

The growth characteristic favored by initially dry followed by moderate stress condition was the seedling emergence, whereas under this treatment the leaf area (a measure of seedling vigour), was greatly reduced when compared with the initial wet followed by moderate stress treatment. The criteria that are normally used in assessing seedling vigour include rate of emergence, seedling height, leaf area, and dry matter accumulation. However, the results of this study seem to indicate emergence can only be an attribute of seedling vigour when moisture is adequate for plant growth. This finding was similar to that of Romo and Haferkamp (1987), that sowing seeds during periods of high soil moisture may give rapid establishment but stimulation of the seeds and seedling populations may be under transient moisture content.

Depending on the level, the moisture stress condition did affect the timing of flowering, since the greatest leaf area, fresh and dry weights were obtained from moderately stressed plants. These plants were more vigorous than both the severely and unstressed treatments. The findings of Adedipe (1976) that moderate stress condition does not bring about a deleterious effect on plant growth seems to buttress the results in this study.

In this investigation, sunflower wilted at -14.6 bar while amaranthus wilted at -23.8 bar. This indicates that amaranthus is more drought tolerant, than sunflower which wilted at >-15.0 bar. In amaranth, the moderate stress condition gave the highest cumulative dry matter production while the no stress condition gave the least. Also, severe and no stress condition decreased seed yield per plant of amaranthus compared to the moderately stressed condition. Findings similar to ours was that of Khatamian et al. (1973) that considering the water use efficiency of the plants, the seed yield under severe and unstressed condition gave $67 \%$ of the moderate stress value.

Conclusively, for the economy of irrigation, the 24/48 hourly watering cycle of -0.3 bar would generally be regarded as over-watering and the results reflect this. Also there is no physiological advantage to be gained from periods of excessive stress (10 to 14 days of 6.0 bar soil water potential). We therefore concluded that amaranthus is drought tolerant and gave optimum growth response when moisture was withheld for 3-4days and soil moisture potential maintained at -1.5 bar to -3.0 bar. However, if the irrigation system breaks down, the crops can still recover provided that the water is unavailable for less than 7 days on a loamy sandy soil with water potential of -6.0 bar.

\section{REFERENCES}

Adedipe. N.O. 1976. Comparative sensitivity of ${ }^{14} \mathrm{C}$ Sucrose and ${ }^{32} \mathrm{P}$ Translocation ofwater stress pulse in Hibiscus esculentus (Okro). Biochem. Physiol. Pflanzen, 169. 347353.

Bhargava, A.. Shikia, S.. Ohri. D.2006. Chenopodium quinoa - An Indian perspective. Industrial crops \& products. 23(1), pp. $73-87$.

David. 0. 0. 2001. Density and cutting height affect production of Celosiu argenteci I.. in the humid tropics. Jour. Vegetable Crop Production, 7(2) pp. 45 - 55.

Food and Agricultural Organization of the United Nations (FAO). 1991. World food statistics, FAO monthly bulletin. FAO, Rome, Italy.

Garcia. M., Raes, D., Jacobsen, S.E. 2003. Evapotranspirational analysis and irrigation requirements of quinoa (Chenopodium quiiwa) in the Bolivian highlands. Agric. Water Mgt.,60pp,119- 134.

Grubben. G.J.H. 1976. The cultivation of amaranth as a tropical leaf vegetable with special reference to $\mathrm{S}$. Dahomey. Printed by Comm. Dept. Agric Res. Royal Trop. Inst. (Netherlands). Comm. 67. 207.

Khatamian, P., Adedipe, N.O., and Armrod, D.P. 1973. Soil aspects of ozone phytotoxicity in tomato plant. Plant and Soil, 38:531-541.

Liu, F.. Stutzel, FI. 2002. Leaf water relations of vegetable 
amaranth (Amuranthus xpp.) in response to soil drying. European Journal of Agronomy, 16 : 137 - 150. Liu. F., Stutzel. H.2004. Biomass partitioning, specified leaf area and water use efficiency of vegetable amaranth (Amaranthus spp.) in response to irrigation frequencies. Scientia Horticulturae, 102 : 5-27.

Mwale, S.S., Azam-Ali, S.N., Massawe, F.J. 2007. Growth and development of bambara groundnut (Vigna subterranean) in response to soil moisture. European Journal of Agronomy, 26 (4) : 354 - 362.

Olufolaji. A.O. and Tayo, T.O. 1980. Growth, development and mineral contents of three cultivals of amaranth.
Scienctia Horticulture, 13:181 - 189.

Olufolaji, A.O. and Tayo, T.O. 1989. The performance of several morphotypes of Amuranthus cruenths L. under two harvesting methods. Trop. Agric. (Trinidad). 66: $273-276$.

Romo, J.T. and Haferkamp, M.R. 1987. Forage kochia germination response to temperature water stress and specific ions. Agron Jour. 79: 27 - 30.

World Health Organization (WHO). 1993. Nutritional Impact of Food on Health. WBO Quarterly Report. Geneva, Switzerland. 